Design/Methods A secondary analysis of prospectively gathered data from a randomised trial of different cord management strategies. 38 preterm infants were monitored between 6 and 18 hours after birth. Echocardiogram measurements of right and left ventricular output(RVO, LVO) and superior vena cava flow(SVC) were performed. cEEG and changes in cerebral blood oxygenation were determined by NIRS at 6 and 12 hour timepoints. Development and grade of IVH was assessed by cranial ultrasound (CRUS) at 24hours. Quantitative features were determined for cEEG and NIRS values. Spearman rank correlations were calculated between RVO, LVO, SVO and the NIRS and EEG quantitative features in infants with and without IVH.

Results Of the 38 infants analysed(median GA 28.0 weeks [23.6-31.6], median BW 950 g [530-2040 g]) 13 preterm infants developed IVH within 24 hours. Following analysis, a notable difference in relations between LVO, RVO and EEG quantitative features was found between those infants with and without IVH. Correlations of EEG features such as IBI Length $\max (r=0.71$, pvalue 0.01$)$, IBI burst $\% \quad(r=-0.61$, pvalue 0.05$)$ and rEEG asymmetry $(r=0.74$, $p$ value 0.01$)$ with LVO showed significant relationships at both $6 \mathrm{hrs}$ and again IBI Length max $(r=0.71$, pvalue 0.01$)$, IBI burst $\% \quad(r=-0.66$, pvalue 0.05$)$ and rEEG asymmetry $(r=0.65$, p value 0.05$)$ at $12 \mathrm{hr}$ timpeoints in the IVH subgroup. No significant correlations were found between NIRS and CO measures in both groups.

Conclusion(s) Correlation of early continuous EEG quantitative data with LVO measures demonstrated a significant difference in features between preterm infants with IVH and those without. These results may indicate that incorporation of $\mathrm{CO}$ measurements and cEEG into a multi-modal method of neonatal monitoring may permit early identification of preterm infants at increased risk of IVH.

\section{OC60 EVALUATION OF PERINATAL DEVELOPMENT OF OFFSPRING BORN TO MOTHERS WITH GESTATIONAL DIABETES}

Irirna Nikitina*, Alisa Masel, Ekaterina Kaprior, Daria Yuablunovskaya, Igor Kelmanson. Almazov National Medical Research Centre, Saint Petersburg, Russian Federation

\subsection{6/archdischild-2019-epa.57}

Background According to The Hyperglycemia and Adverse Pregnancy Outcome Study (HAPO Study) 2008, hyperglycemia caused by maternal gestational diabetes mellitus (GDM) is an important contributor to adverse fetal programming and maternal complications.

Aim To assess the associations between the GDM and perinatal outcomes.

Design The study comprised 228 women who were divided into two groups: Group 1 (Gr1) included 157 women with GDM, group 2 (Gr2) - 71 women without GDM. The diagnosis of GDM has been confirmed according to the HAPO criteria. Retrospective analysis of pregnancy, delivery and early neonatal period were performed. Newborn anthropometric characteristics were assessed and interpreted according to INTERGROWTH-21 ${ }^{\text {st }}$ recommendations. Statistical significance was estimated using Mann-Whitney $U$ and chisquared tests.

Results The rate of vaginal deliveries in both groups was similar (Gr1 81.7\%; Gr2 73.5\%, p = 0.19). The presence of
GDM was associated with an increased risk of obstetric injuries (OR 4.1, 95\% CI 1.9-8.5; p = 0.00004) and hypoglyce$\mathrm{mia}$ in the newborn (OR 5.4, 95\% CI 2.2-13.1; $\mathrm{p}=$ 0.00002). No significant differences were found in birth weight (Gr1: Me $3500 \pm 400 \mathrm{~g}$ vs Gr2: Me $3450 \pm 500 \mathrm{~g}$, $\mathrm{p}=0.7$ ), birth length (Gr1: Me $52 \pm 0.2 \mathrm{~cm}$ vs Gr2: Me $52 \pm 0.2 \mathrm{~cm}, \mathrm{p}=0,3$ ), weight/length ratio (Gr1: Me $6.7 \pm$ $0.5 \mathrm{~kg} / \mathrm{cm}$ vs Gr2: Me $6.6 \pm 0.7 \mathrm{~kg} / \mathrm{cm}, \mathrm{p}=0.9)$ and head circumference (Gr1: Me $35.2 \pm 2.3 \mathrm{~cm}$ vs Gr2: Me $35.0 \pm$ $1.3 \mathrm{~cm}, \mathrm{p}=0.05)$. The risk of being born with 'upper average' weight was associated with presence of maternal GDM ( $>1$ SDS $<2$ SDS) (OR 2.47; 95\% CI 1.2-5.1; p $=0.01)$. However, no association was found between GDM and neonatal weight exceeding 2 SDS, known as 'macrosomia' (OR 1.1; 95\% CI $0.3-3.7 ; p=0.83)$. Maternal GDM was also associated with increased risk of neonatal head circumference in the 'upper average' range (> 1 SDS) (OR 2.4; 95\% CI 1.4-5.9; $\mathrm{p}=0.005)$.

Conclusion Maternal GDM was associated with increased risk of obstetric injuries, which can be explained by higher rates of increased newborn weight and head circumference. However, there was no increased number of children with macrosomia born to GDM mothers, which indicates a limited impact of GDM on fetal development compared to diabetes type 1 or 2 . Neonatal hypoglycemia was associated with maternal GDM as well.

\section{OC61 A SYSTEMATIC REVIEW OF SERUM BIOMARKERS IN NEONATAL ENCEPHALOPATHY TO PREDICT SHORT TERM OUTCOME}

1,2,3Mary O'Dea*, ${ }^{1}$ Tim Hurley, ${ }^{4}$ David Mockler, ${ }^{5}$ Kasper Kyng, ${ }^{6}$ Phillips Bob, 2,7,8,9Eleanor Molloy. ${ }^{1} 1$. Department of Paediatric and Child Health Trinity College Dublin, Dublin, Ireland; ${ }^{2}$ Coombe Women and Infant's University Hospital, Dublin, Ireland; ${ }^{3}$ National Children's Research Centre, Crumlin, Dublin, Ireland; ${ }^{4} 2$. Subject Librarian, School of Medicine, Trinity College Dublin, Dublin, Ireland; ${ }^{5}$. University of Aarhus, Aarhus, Denmark; ${ }^{6} 4$. University of York, York, UK; ${ }^{7}$ Department of Paediatric and Child Health Trinity College Dublin, Dublin, Ireland; ${ }^{8}$ Our Lady's Childrens Hospital, Crumlin, Dublin, Ireland; ${ }^{9}$ National Children's Hospital, Tallaght, Dublin, Ireland

\subsection{6/archdischild-2019-epa.58}

Background Neonatal Encephalopathy (NE) describes central nervous system dysfunction from all causes and has a multifactorial aetiology. NE is difficult to diagnose, to treat and to predict outcome. Early prognostic information is important to initiate early intervention of therapies, to counsel parents and to redirect care. There is no gold standard early biomarker to predict outcome at present.

Methods The review protocol was prospectively registered with Prospero. A comprehensive search with relevant search terms of online databases Em Base, PubMed, Cochrane and Web of Science for Biomarkers in Neonatal Encephalopathy to Predict outcome was performed. The short term outcomes examined include severity of Neonatal Encephalopathy, MRI brain and survival in the neonatal period.

Two independent reviewers used Covidence software to select the studies. Quality assessment was performed using Cochrane Collaboration Tool for Risk of Bias Assessment and quantitative analysis was completed using Revman software (5.3).

Results and Discussion 1613 papers were identified after duplicates were excluded, and narrowed to 314 papers following abstract review for full text examination. Following full text 